\title{
Neoteric FT-IR Investigation on the Functional Groups of Phosphonium- Based Deep Eutectic Solvents
}

\section{Tayeb Aissaoui ${ }^{1 *}$ and Inas M AINashef ${ }^{2}$}

${ }^{1}$ Department of Chemical Engineering, University of Technology Petronas, 31750 Tronoh, Perak, Malaysia

${ }^{2}$ Department of Chemical and Environmental Engineering, Masdar Institute of Science and Technology, Abu Dhabi, United Arab Emirates

\begin{abstract}
Deep eutectic solvents (DESs) are novel solvent media that are currently under investigation as an alternative to ionic liquids and conventional solvents. The physical properties of DESs as well as their mild environmental footprint and potentially critical industrial application necessitate understanding the interaction of functional groups on both the salt and hydrogen bond donor (HBD). In this study, four DESs were prepared by mixing triethylenglycol, diethylenglycol, ethylenglycol, and glycerol as HBDs with methyltriphenyl phosphonium bromide as a salt at a molar ratio of 1:4. Fourier transform infrared spectroscopy was conducted to highlight the chemical structure and mechanism of the combination of the four DESs. New spectra illustrating the combination of the functional groups of the HBDs and salt were observed and interpreted. This study is the first to investigate the properties of neoteric FT-IR for phosphonium-based DESs in addition to (MTPB:TEG) which was reported previously.
\end{abstract}

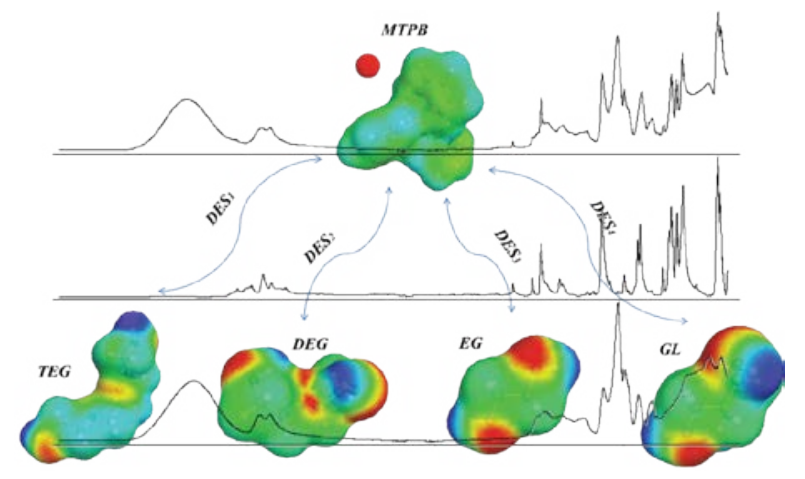

Keywords: Deep eutectic solvents; Ionic liquid; Green chemistry; Phosphonium salts; Fourier transform infrared spectroscopy

\section{Introduction}

In recent decades, deep eutectic solvents (DESs) have emerged as a novel class of ionic liquids (ILs) [1]. DESs share many characteristics with conventional ILs (e.g., no flammability and low volatility) [2]. In addition, DESs are relatively cheap to produce because they are made from inexpensive starting materials and are easy to prepare. Moreover, biodegradable and nontoxic constituents can be employed to prepare DESs [3].

DESs are a combination of two or more compounds, and the final solvent has a melting point that is lower than that of its individual components. DESs comprise mixtures of organic halide salts, such as choline chloride $(\mathrm{CHCl})$, with an organic compound that acts as a hydrogen bond donor (HBD). The HBD can form a hydrogen bond with the halide ion. Examples of HBDs include amides, amines, alcohols, and carboxylic acids [4]. By convention, DESs must have a melting point equal to or less than $100^{\circ} \mathrm{C}$.

Recently, Kareem et al. (2010), Luo et al. (2012), Shahbaz et al. (20112012) and Hayyan et al. (2013) have reported the physical properties of both phosphonium- and ammonium-based DESs and demonstrated their potential use in industrial applications [5-11]. Hayyan et al. (2013) analyzed the cytotoxicity and toxicity of phosphonium- and ammonium-based DESs [12].
In the present study, four DESs were prepared by mixing triethylenglycol (TEG), diethylenglycol (DEG), ethylenglycol (EG), and glycerol (GL) as HBDs with methyltriphenylphosphonium bromide (MTPB) as a salt at a molar ratio of 1:4. Fourier transform infrared spectroscopy (FT-IR) was performed for each DES to compare the components with their products and identify the transformation resulting from the formation of the DESs.

\section{Materials and Method}

\section{Chemicals}

MTPB (99\% purity) and TEG (99\% purity) were obtained from Merck Chemicals (Darmstadt, Germany). DEG, EG, and GL (99.5\% purity) were obtained from R\&M Chemicals Ltd. (Essex, UK). Table 1

*Corresponding author: Tayeb Aissaoui, Department of Chemical Engineering, University of Technology Petronas, 31750 Tronoh, Perak, Malaysia, Tel: +6-0183197431; E-mail: t.aissaoui84@gmail.com

Received October 31, 2015; Accepted November 23, 2015; Published November 26, 2015

Citation: Aissaoui T, AINashef IM (2015) Neoteric FT-IR Investigation on the Functional Groups of Phosphonium-Based Deep Eutectic Solvents. Pharm Anal Acta 6: 449. doi:10.4172/21532435.1000449

Copyright: (c) 2015 Aissaoui T, et al. This is an open-access article distributed under the terms of the Creative Commons Attribution License, which permits unrestricted use, distribution, and reproduction in any medium, provided the original author and source are credited. 
Citation: Aissaoui T, AINashef IM (2015) Neoteric FT-IR Investigation on the Functional Groups of Phosphonium-Based Deep Eutectic Solvents. Pharm Anal Acta 6: 449. doi:10.4172/21532435.1000449

shows the salt, $\mathrm{HBD}$, abbreviations, molar ratio, and symbols of the four DESs. The chemicals were stored in a highly controlled environment to prevent moisture and impurity contamination.

\section{DES preparation}

The DESs were prepared according to methods reported in the literature [1]. In brief, a measured amount of MTPB was mixed with one of the four HBDs (TEG, DEG, EG, and GL) at a molar ratio of 1:4 and placed in an incubator shaker (Brunswick Scientific Model Innova $40 \mathrm{R}$ ). The mixture was shaken at $350 \mathrm{rpm}$ at a temperature of $80^{\circ} \mathrm{C}$ for 2-3 hours until the DESs became homogeneous with no precipitation.

\section{FT-IR analysis}

A Spectrum 400 FT-IR spectrometer was employed to analyze the functional groups of the four DESs at room temperature. The four HBDs (TEG, DEG, EG, and GL) and the salt, MTPB were analyzed using FT-IR to identify the transformations resulting from the formation of the DESs.

\section{Results and Discussion}

The chemical structures and combination mechanism of the DESs are unknown and were therefore investigated in this study. It should be noted that DES (MTPB:TEG) FT-IR analysis was investigated by Hayyan et al. (2015) [13] and repeated in this article for comparison. The observed FT-IR spectra revealed the formation of proton transfer salts. The presence of $\mathrm{O}-\mathrm{H}$ stretching bands between 3200 and 3500 $\mathrm{cm}^{-1}$ in the four spectrum figures was attributed to hydroxyl telescopic vibrations at $3300 \mathrm{~cm}^{-1}[6,14-16]$. Figure 1 reveals that a shift in the $\mathrm{OH}$ stretching vibration of pure ethyleneglycol occurred when the DES was formed. This in agreement with the observation reported by Hou et al. for a DES formed using levulinic acid as the HBD and tetrabutylphosphonium bromide [17]. The FT-IR spectra of the TEG in the DES indicate that the change in vibrational state occurred because a portion of the cloud of electrons of the oxygen atom was transferred to the hydrogen bond, reducing the force constant. Thus, the shift of the $\mathrm{OH}$ stretching vibration indicated the existence of a hydrogen bond between the TEG and TMPB when the DES was formed. The region between 3000 and $2800 \mathrm{~cm}^{-1}$ shows the existence of $\mathrm{C}-\mathrm{H}$ stretching bands of the alkanes $\mathrm{CH}_{3}$ and $\mathrm{CH}_{2}$ for the four DESs [6,14-16]. Because of the water content and organic structure of the HBDs (i.e., TEG, DEG, EG, and GL) and organic salt (i.e., MTPB), the functional groups of the four formed DESs appear to be relatively consistent. For the phosphonium-based DESs (i.e., $\mathrm{DES}_{1}, \mathrm{DES}_{2}, \mathrm{DES}_{3}$, and $\mathrm{DES}_{4}$ ), the $\mathrm{P}-\mathrm{H}$ stretching bands may be overlapped with $\mathrm{C}-\mathrm{H}$ vibrational bands in the region of 3000-2800 $\mathrm{cm}^{-1}$ at frequencies lower than $3000 \mathrm{~cm}^{-1}$.

Comparing Figures 1-4 shows that the stretching vibration in the region of $2000-3100 \mathrm{~cm}^{-1}$ in the MTPB disappeared after the phosphonium-based DESs were formed. For the four DESs, the region of $2340-4000 \mathrm{~cm}^{-1}$ contains only two peaks, proving the existence of an $\mathrm{OH}$ group and phosphonium cation.

The presence of $\mathrm{Br}$ in the formed DESs was also observed in the region of $600-408 \mathrm{~cm}^{-1}$ (Figures 1-4) [14-16]. The four DESs

\begin{tabular}{|l|c|c|c|c|}
\hline \multicolumn{1}{|c|}{ Salts } & Abbreviation & HBD & Molar ratio & Symbol \\
\hline Methyltriphenylphosphonium bromide & MTPB & TEG & $1: 4$ & $\begin{array}{c}\mathrm{DES}_{1} \\
{[13]}\end{array}$ \\
\hline Methyltriphenylphosphonium bromide & MTPB & DEG & $1: 4$ & $\mathrm{DES}_{2}$ \\
\hline Methyltriphenylphosphonium bromide & MTPB & EG & $1: 4$ & $\mathrm{DES}_{3}$ \\
\hline Methyltriphenylphosphonium bromide & MTPB & GL & $1: 4$ & DES $_{4}$ \\
\hline
\end{tabular}

Table1: Composition and abbreviation of DESs used in this research.

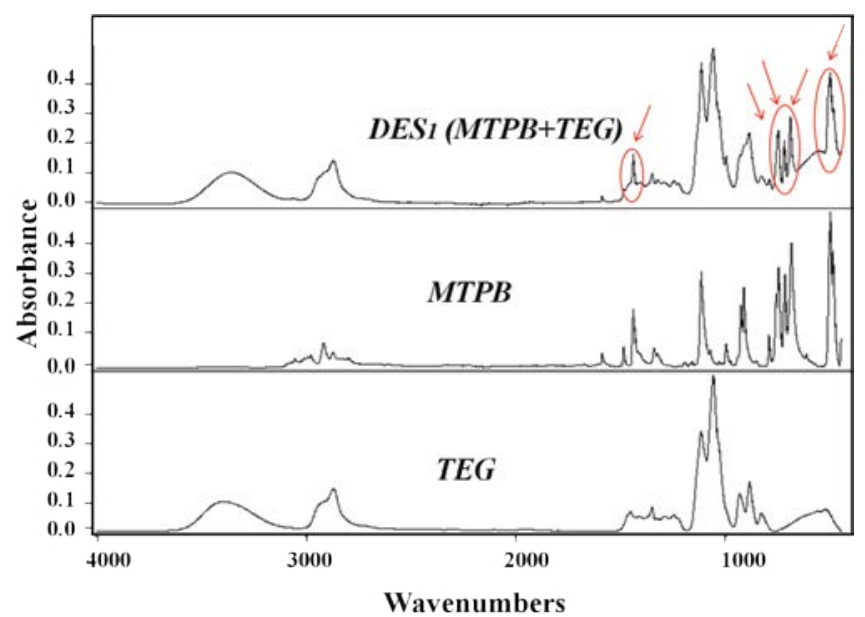

Figure 1: FT-IR spectra of the TEG, MTPB and DES , $_{1}$ at room temperature in the region of $400-4000 \mathrm{~cm}^{-1}$ [13]

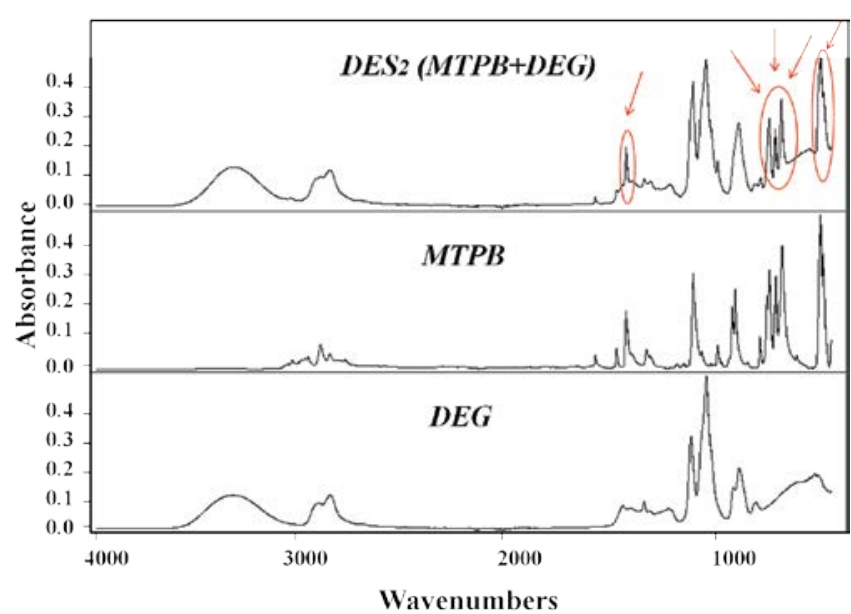

Figure 2: FT-IR spectra of the DEG, MTPB and DES ${ }_{2}$ at room temperature in the region of $400-4000 \mathrm{~cm}^{-1}$.

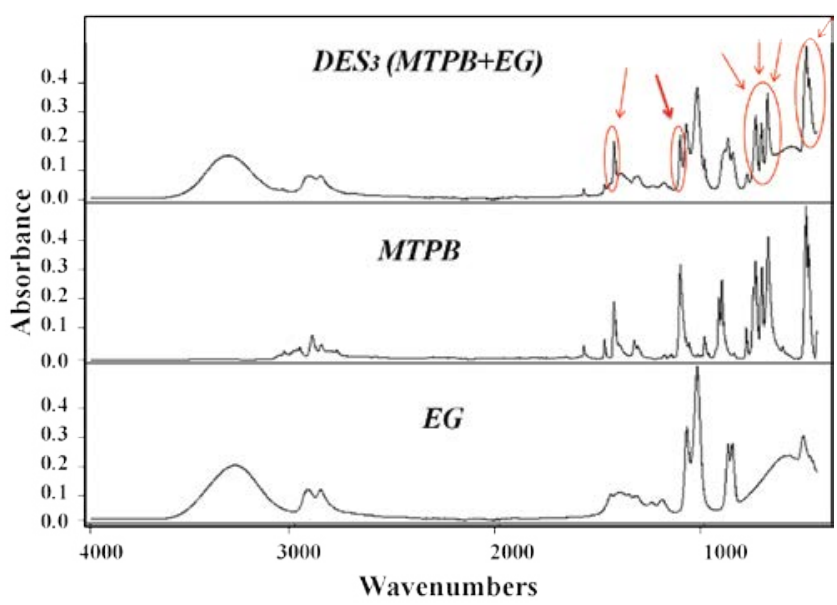

Figure 3: FT-IR spectra of the EG, MTPB and DES ${ }_{3}$ at room temperature in the region of $400-4000 \mathrm{~cm}^{-1}$. 
Citation: Aissaoui T, AINashef IM (2015) Neoteric FT-IR Investigation on the Functional Groups of Phosphonium-Based Deep Eutectic Solvents. Pharm Anal Acta 6: 449. doi:10.4172/21532435.1000449

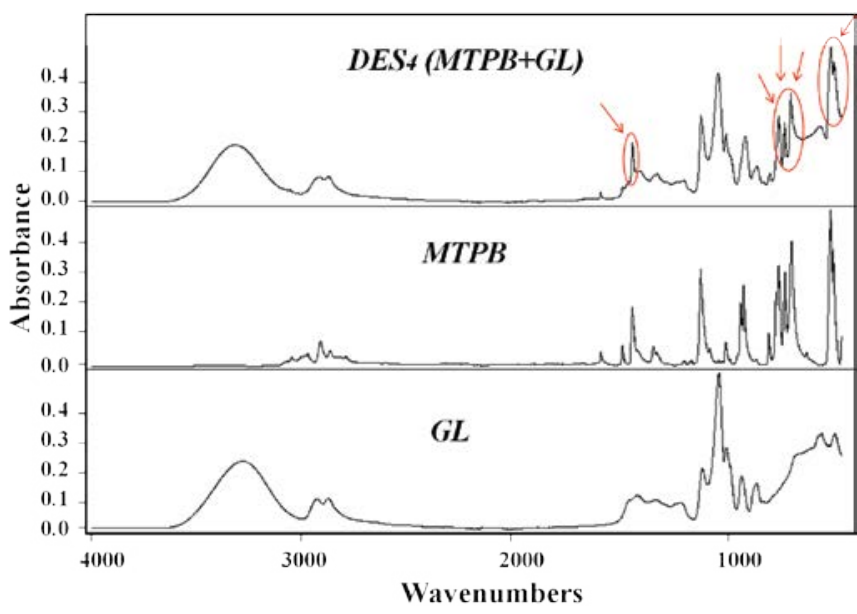

Figure 4: FT-IR spectra of the GL, MTPB and DES 4 at room temperature in the region of $400-4000 \mathrm{~cm}^{-1}$.

demonstrate similar chemical compositions for $\mathrm{DES}_{1}, \mathrm{DES}_{2}, \mathrm{DES}_{3}$, and $\mathrm{DES}_{4}$ with varying levels of absorbance. In addition, a peak not present in the other three DES spectra was observed at $1119 \mathrm{~cm}^{-1}$ in $\mathrm{DES}_{3}$.

To the best of our knowledge, few studies have analyzed the functional groups of phosphonium-based DESs. Therefore, we recommend that future studies on DESs conduct further analyses.

\section{Conclusion}

In this FT-IR investigation, the functional groups of four neoteric phosphonium DESs were analyzed. The findings indicate that the functional groups of the mixed DESs behave as their HBDs, and the appearance of new peaks confirms the phosphonium identity of the DESs. The findings of this study warrant further investigation of DES green solvents to elucidate the molecular structure and combination mechanism of DESs.

\section{Acknowledgement}

The authors of this article would like to express their thanks to University of Malaya Centre for lonic Liquids (UMCiL) where the experiments were conducted.

\section{References}

1. Mandroyan A, Mourad-Mahmoud M, Doche ML, Hihn JY (2014) Effects of Ultrasound and Temperature on Copper Electro Reduction in Deep Eutectic Solvents (Des). Ultrason Sonochem 21: 2010-2019.
2. Carriazo D, Serrano MC, Gutiérrez MC, Ferrer ML, del Monte F (2012) DeepEutectic Solvents Playing Multiple Roles in the Synthesis of Polymers and Related Materials. Chem Soc Rev 41: 4996-5014.

3. Maugeri Z, de María PD (2014) Benzaldehyde Lyase (Bal)-Catalyzed Enantioselective Cc Bond Formation in Deep-Eutectic-Solvents-Buffer Mixtures, Journal of Molecular Catalysis B: Enzymatic 107: 120-123.

4. Hayyan M, Mjalli FS, Hashim MA, AINashef IM (2010) A Novel Technique for Separating Glycerine from Palm Oil-Based Biodiesel Using lonic Liquids. Fuel Processing Technology 91: 116-120.

5. Kareem MA, Mjalli FS, Hashim MA, AINashef IM (2010) Phosphonium-Based Ionic Liquids Analogues and Their Physical Properties. J Chem Eng Data 55: 4632-4637.

6. Luo J, Conrad O, Vankelecom IFJ (2012) Physicochemical Properties of Phosphonium-Based and Ammonium-Based Protic Ionic Liquids, Journal of Materials Chemistry 22: 20574-20579.

7. Shahbaz K, Mjalli FS, Hashim MA, AINashef IM (2011) Eutectic Solvents for the Removal of Residual Palm Oil-Based Biodiesel Catalyst. Separation and Purification Technology 81: 216-222.

8. Shahbaz K, Baroutian S, Mjalli FS, Hashim MA, AINashef IM (2012) Densities of Ammonium and Phosphonium Based Deep Eutectic Solvents: Prediction Using Artificial Intelligence and Group Contribution Techniques. Thermochimica Acta 527: 59-66.

9. Shahbaz K, Mjalli FS, Hashim MA, AINashef IM (2012) Prediction of the Surface Tension of Deep Eutectic Solvents. Fluid Phase Equilibria 319: 48-54.

10. Shahbaz K, Mjalli FS, Hashim MA, AINashef IM (2011) Prediction of Deep Eutectic Solvents Densities at Different Temperatures. Thermochimica Acta 515: 67-72.

11. Hayyan A, Hashim MA, Mjalli FS, Hayyan M, AINashef IM (2013) A Novel Phosphonium-Based Deep Eutectic Catalyst for Biodiesel Production from Industrial Low Grade Crude Palm Oil. Chemical Engineering Science 92: 81-88.

12. Hayyan M, Hashim MA, Al-Saadi MA, Hayyan A, AINashef IM (2013) Assessment of Cytotoxicity and Toxicity for Phosphonium-Based Deep Eutectic Solvents. Chemosphere 93: 455-459.

13. Hayyan M, Aissaoui T, Hashim MA, AISaadi MA, Hayyan A (2015) Triethylene glycol based deep eutectic solvents and their physical properties. Journal of the Taiwan Institute of Chemical Engineers 50: 24-30.

14. Stuar BH (2004) Infrared Spectroscopy: Fundamentals and Applications, John Wiley and Sons, Ltd, England.

15. Roeges NPG (1994) A Guide to the Complete Interpretation of Infrared Spectra of Organic Structures, John Wiley and Sons Ltd, England.

16. Smith BC (1999) Infrared Spectral Intepretation: A Systematic Approach, CRC press LLC, USA.

17. Hou Y, Li Z, Ren S, Wu W (2014) Separation of toluene from toluene/alkane mixtures with phosphonium salt based deep eutectic solvents. Fuel Processing Technology 135: 99-104. 